TRABAJO EN PROGRESO

\title{
Hacia un sistema parlante para personas ciegas y de baja visión
}

\author{
Yusmar Alexis Flores Alvarado, Nieves Jiménez Mía, \\ Antonio Arriaga Fierro, María del Rosario Peralta \\ Calvo, Zulma Janet Hernández Paxtián
}

Publicado: 31 Octubre 2018

\begin{abstract}
Resumen
La tecnología está creciendo de forma exponencial, esto está causando avance en diversas áreas. Además, muchas tareas que se hacían de forma personal y presencial, que implicaba gastos, tiempo y otras cuestiones, ahora pueden hacerse por medio de la tecnología. Sin embargo, se ha formado una brecha entre la tecnología y las personas de capacidades diferentes. Este proyecto se enfoca en personas ciegas, tiene como fin la implementación de un asistente en la enseñanza de tecnologías [1]. En este reporte se presenta el proceso que llevó trabajar sobre la implementación de este sistema parlante para personas ciegas y de baja visión. Se plantea desde el análisis realizado, diseño del sistema, pruebas realizadas y los resultados obtenidos de los prototipos generados.
\end{abstract}

Palabras clave: Personas ciegas, UCD, Pruebas de Usabilidad, Diseño de interacción.

\section{Introducción}

Según el INEGI, en el año 2010 el número de personas ciegas en México era de 1,561,081; en Oaxaca era 65244 [4]. En Teotitlán de Flores Magón no hay registro respecto al número de personas ciegas, lo cual es un problema porque eso hace ver la poca importancia sobre este grupo de personas vulnerables. Con base a un estudio contextual se encontró que existe una gran brecha entre la tecnología y las personas débiles visuales a causa de que no hay forma de que aprendan a menos que una persona les dedique tiempo y les enseñe a utilizar la tecnología, pues los dispositivos están a su alcance. Sistema Diseñado para Ayudar, Motivar y Mejorar la Experiencia de las Personas Débiles Visuales con la Tecnología (SAMM por sus siglas en inglés) [1] está planeado para ser el medio por el que principalmente los niños ciegos puedan tener el primer contacto con la tecnología. SAMM sería un asistente en la enseñanza de las tecnologías, interactuaría amigablemente con el usuario para que de forma eficaz aprenda a adentrarse en la tecnología. SAMM se centra en enseñar de forma

Flores Alvarado YA., Jiménez Mía N., Arriaga Fierro A, Peralta Calvo

MR, Hernández Paxtian ZJ

Universidad de la Cañada

Teotitlán de Flores Magón, Oaxaca, México

Email: li_yusmar@unca.edu.mx,li_nievesjm@unca.edu.mx,

li_antonioaf@unca.edu.mx,mperalta@unca.edu.mx,

jpaxtian@unca.edu.mx eficaz a utilizar la computadora, es una buena opción porque va a guiarlo en todo momento, se va a convertir en un tutor, el cual va a motivar y mejorar la experiencia al aprender. Por el momento el sistema se enfoca en la tarea de reconocer el teclado, esto debido a que una vez que el usuario aprenda a utilizar el teclado, por consecuencia usar una computadora será sumamente fácil.

\section{Metodología}

SAMM se desarrolla bajo la metodología Diseño Centrado en el Usuario (por sus siglas en inglés, UCD) [6]. Con la finalidad de establecer un marco de trabajo para el desarrollo de SAMM en equipo, se recurrió a la metodología ágil de SCRUM [7]. Esto permite poder hacer mejoras en los prototipos que se van entregando a los usuarios, y personas que apoyan a débiles visuales y ciegos.

\section{Fase de Requerimientos}

En el primer ciclo de la metodología UCD se obtuvo un prototipo funcional de SAMM, éste contemplaba la función de asistir al usuario a reconocer el teclado, a través de la interacción con el sistema parlante. Se realizaron pruebas de Usabilidad, en el UsaLab (Labotatorio de usabilidad perteneciente a la Universidad Tecnológica de la Mixteca), se realizaron 2 pruebas con usuarios de baja visión (Ver Figura 1). Las tareas que conformaban el sistema eran las siguientes. Nombre de las teclas: SAMM guía al usuario por medio de instrucciones a presionar las teclas, cuando es presionada, SAMM menciona su nombre. Funciones de las teclas: SAMM guía al usuario a presionar solo las teclas que cuentan con funciones y les menciona su funcionamiento de forma sencilla. Partes del teclado: SAMM guía al usuario a presionar teclas de grupo en grupo, mencionando su nombre y enfatizando su funcionamiento. Los resultados obtenidos se muestran en la Tabla 1, 2 y 3. Parte de la base de los requerimientos fueron las pruebas que se hicieron para evaluar el funcionamiento del primer prototipo funcional

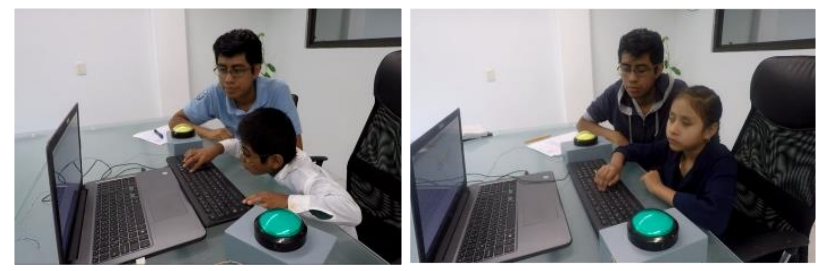

Figura 1. Pruebas de Usabilidad 
Tabla 1. Resultado obtenido de la suma de efectividades

\begin{tabular}{|c|c|}
\hline Estudio realizado & $\mathbf{1}$ \\
\hline Pruebas realizadas & 2 \\
\hline Tareas realizadas & 6 \\
\hline Tareas exitosas & 4 \\
\hline Efectividad promedio & $66.6 \%$ \\
\hline
\end{tabular}

Tabla 2. Promedio de la eficiencia del sistema

\begin{tabular}{|c|c|}
\hline Tarea & $\begin{array}{c}\text { Tiempo } \\
\text { Promedio }\end{array}$ \\
\hline Nombre de las teclas & $6 \mathrm{~min}$ \\
\hline Funciones de las teclas & $7.5 \mathrm{~min}$ \\
\hline Partes del teclado & $9 \mathrm{~min}$ \\
\hline
\end{tabular}

Tabla 3. Satisfacción del usuario. Opiniones de los usuarios a las preguntas. ¿qué no te gustó? y ¿con cuanto evalúas al sistema?

\begin{tabular}{|c|c|c|}
\hline Usuario & Tarea & Comentario \\
\hline 1 & 1 & "El ruido que hizo" \\
\hline 2 & & "Cuando habla muchas veces" \\
\cline { 1 - 1 } & 2 & Nada \\
\hline 2 & & Diez \\
\cline { 1 - 1 } & & \\
\cline { 1 - 2 } & &
\end{tabular}

Tabla 4. Perfil de usuario

\begin{tabular}{|c|l|}
\hline Usuario & \multicolumn{1}{|c|}{ Características } \\
\hline $\begin{array}{c}\text { Niños de 6 } \\
\text { a 10 años }\end{array}$ & $\begin{array}{l}\text { Pérdida de visión total o parcial } \\
\text { Puede o no tener experiencia con una } \\
\text { computadora } \\
\text { Sexo indistinto }\end{array}$ \\
\hline
\end{tabular}

Tabla 5. Funciones de las teclas en cada escenario

\begin{tabular}{|c|c|c|}
\hline Usuario & Tarea & Comentario \\
\hline Escuela & Retroceso & $\begin{array}{c}\text { Salir de la escuela y entrar al } \\
\text { escenario general }\end{array}$ \\
\hline \multirow[t]{2}{*}{ General } & Dirección & Avanzar a respectiva dirección \\
\hline & Enter & Entrar a algún establecimiento \\
\hline \multirow[t]{4}{*}{ Tiendas } & Numéricas & $\begin{array}{l}\text { Introducir cantidad de precio } \\
\text { para determinado producto }\end{array}$ \\
\hline & Enter & Comprar el producto \\
\hline & Retroceso & $\begin{array}{c}\text { Salir de establecimiento y entrar } \\
\text { al escenario general }\end{array}$ \\
\hline & Tabulador & Seleccionar otro producto \\
\hline
\end{tabular}

Si bien, el número de usuarios como se indica en la tabla 2 , no cumple con el mínimo indicado por Jacok Nielsen [5] en las pruebas de usabilidad realizadas, los resultados son relevantes para continuar con un siguiente ciclo de la metodología UCD puesto que ambos usuarios se emocionaron al interactuar con un sistema así, el sentir una voz virtual interactuando con ellos en lugar de una persona, respondieron bien al sistema, pero era tedioso, no era muy entretenido, sin embargo, cuando se les evaluó algunas teclas contestaron bien. Para dicho ciclo se tendrán en cuenta las mejoras que los usuarios dieron a conocer, al igual que las siguientes observaciones que los directivos y profesores de Centros de Atención Múltiple y USAER, quienes estuvieron durante la prueba: La voz del sistema parlante en los audios debe ser amigable al usuario, la retroalimentación auditiva debe ser breve y clara, la narrativa debe ser utilizando analogías que se adecuen al contexto del usuario para la fácil comprensión. Con base a lo anterior, la Tabla 4, indica el perfil del usuario para el siguiente ciclo iterativo del proyecto SAMM.

Analizando las mejoras, se determina que es necesario reforzar la explicación del sistema parlante en el primer escenario de interacción con el usuario, el cual involucra el reconocimiento del teclado. Para esto en una revisión bibliográfica y verificando con los especialistas del CAM 21, se utilizan técnicas que permitan la descripción clara y precisa para reconocer objetos. Se deben tomar en cuenta cuatro conceptos para el desarrollo cognitivo de los niños invidentes como videntes: esquema, asimilación, acomodación y equilibrio [2]. Por otro lado Debra Levin Gelman [2] menciona que los métodos de enseñanza tradicionales no son lo contrario a los juegos, puesto que se puede aprovechar un juego para que los usuarios aprendan. De esta manera, es que se ha incluido como parte de SAMM un juego que apoye a este escenario inicial y de gran importancia para lo que se derivará en la interacción con la computadora por parte del usuario.

\section{Fase de Diseño}

Con base a un estudio contextual e investigación realizada sobre personas invidentes que saben utilizar la computadora, se percibió que las teclas indispensables para ese uso, fueron las teclas: tabulador, enter, retroceso, las teclas alfabéticas y los números. El juego fue diseñado de tal forma que el usuario utilice las cuatro direcciones básicas. En la Tabla 5 se muestran las funciones que tienen las teclas dependiendo del escenario en donde se encuentre el usuario. Es importante mencionar que el juego está enfocado dando prioridad a interfaces auditivas debido al perfil del usuario.

Stephanidis Constantine [8] menciona que por el surgimiento del diseño centrado en el usuario se desarrollaron varios métodos y técnicas de diseño. En este proyecto se utilizaron varias, entre ellas, los Storyboard, Estos con el fin de generar ejemplos de cómo el usuario estaría realizando tareas específicas en un contexto específico con el sistema a desarrollar, y también para comprender y aclarar los requisitos del usuario.

En la Figura 2 se muestra un Storyboard del prototipo. En la Figura 2a se marca el inicio de la interacción entre el usuario y el sistema, donde SAMM se presenta y da a conocer las acciones que podrá realizarse con su interacción. La estructura de SAMM es semejante a la planteada en el artículo [1]. En la Figura 2b se comienza el juego con lo que el sistema parlante de SAMM se apoyará en la interacción con el usuario para reconocer el teclado de la computadora. Nótese que se encuentra un icono de ubicación dentro del juego, tipo avatar, este será el que representará al usuario en el juego.

El diseño del juego representa el contexto del recorrido que seguiría el niño de ir camino de la escuela a casa, donde el usuario por medio de las teclas conducirá al personaje virtual a través del camino diseñado, como se muestra en la Figura 2c. El camino comienza en la escuela y termina en la casa. En el transcurso el 


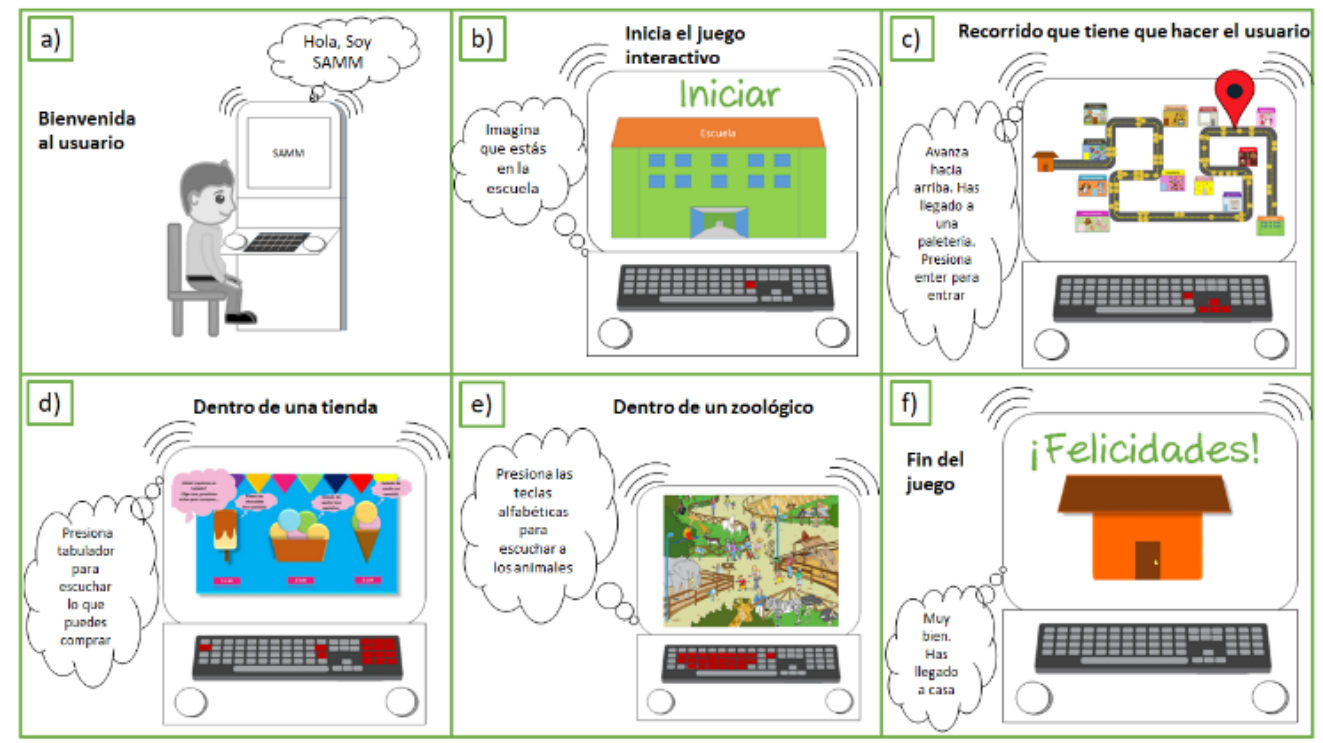

Figura 2. Storyboard del prototipo.

personaje podrá acceder a los establecimientos que están de paso en el camino. En la Figura 3 se muestra de forma más clara el escenario general.

Los establecimientos representan los lugares que pueden estar en el recorrido del niño y que sean de su interés, como se muestra en la Figura 2d. Tal es el caso de algún establecimiento donde pueda comprar un producto. Hassenzahl Marc [3] menciona que todo lo que se desarrolle se debe hacer con el fin de causar emociones en los usuarios. El escenario que se muestra en este inciso considera elementos que llaman la atención a los niños como los animales, la música, dulces, etc. De igual forma la idea del recorrido que tiene que hacer para ir a su casa. Además se planteó la idea de que conforme avanzara hacia su casa, fuese encontrando monedas para que en un momento dado al encontrar una tienda pudiese comprar productos llamativos al usuario. Dicha moneda fue llamada "sammachi", siendo una moneda falsa pero ilustrativa a los usuarios.

Un establecimiento es un zoológico (Figura 2e) donde el usuario podrá interactuar con las teclas alfabéticas para poder conceptualizarlas con ayuda de sonidos de animales que comiencen con estas letras, es decir, cada tecla tendrá el audio de un animal correspondiente a la tecla alfabética pulsada

El escenario en donde el usuario llega al punto final del juego (Figura 3f), retroalimenta al usuario, indicando que ha llegado a la meta.

Con base a los diseños se realizaron prototipos de alta fidelidad, siendo unos wireframe hechos en computadora. Aunado a esto se establecieron los mensajes con que el sistema estaría retroalimentando al usuario en función al escenario en donde se encuentre en el juego.

\section{Evaluación}

Se realizaron Pruebas de Mago de Oz [9] con el prototipo implementado como se muestra en la Figura 4. Dichas pruebas se hicieron tomando en cuenta la tarea de reconocimiento del teclado. En este caso el mago de Oz simuló la voz de SAMM a partir de los mensajes establecidos para cada escenario. El perfil de usuario de SAMM para esta prueba fue sustituido por el especialista en Educación Especial, que apoyaría en la validación de cómo SAMM interactúa con el niño ciego. De esta prueba se obtuvieron los siguientes comentarios: El juego es una forma adecuada para que los niños con estas capacidades conozcan la computadora. El juego es sencillo, la retroalimentación es clara. El juego es entretenido, de forma indirecta el niño estaría aprendiendo cómo es el teclado. Existen aspectos a mejorar respecto a cómo ubicar al niño en la tecla que debe presionar. También se debe retroalimentar un poco al niño sobre lo que está sucediendo en el juego.

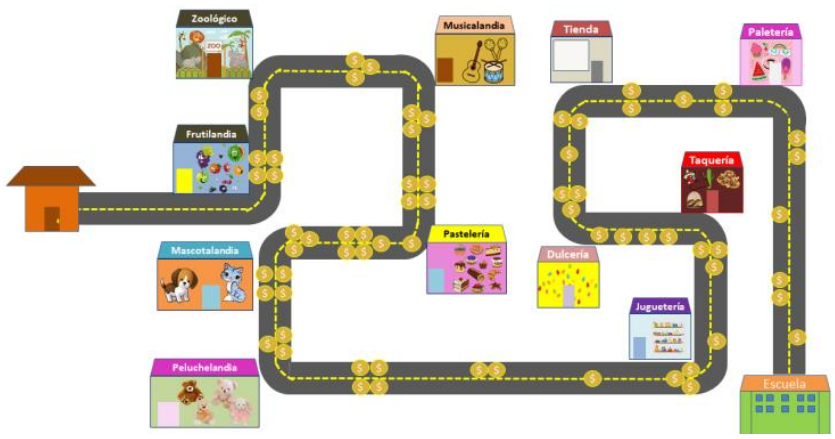

Figura 2. Escenario general del juego diseñado
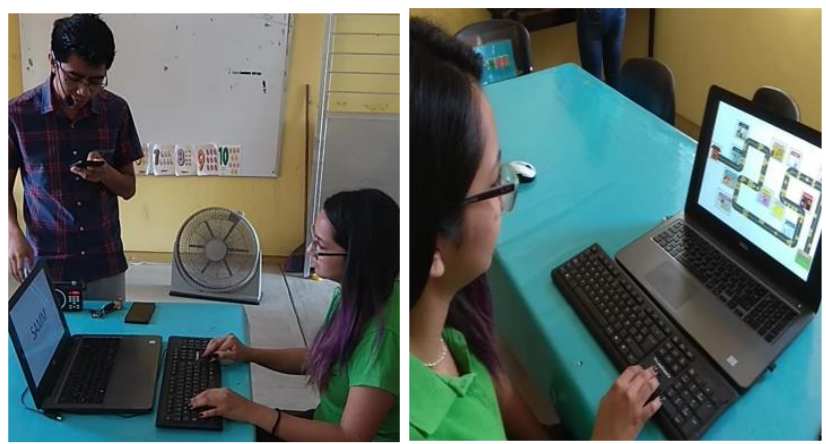

Figura 3. Pruebas de Mago de $\mathrm{Oz}$ 


\section{Conclusión}

SAMM es una buena opción para comenzar a introducir a los niños ciegos con la tecnología. Las pruebas de usabilidad realizadas permiten detectar mejoras por hacer en el sistema. Lo que se lleva hasta este momento de SAMM ayudará al usuario en la tarea de reconocer el teclado. A comparación del primer prototipo presentado en la fase de requerimientos, en este prototipo se buscó la forma de presentar al usuario una forma de aprendizaje entretenida, aplicando analogías a su edad para mejorar el aprendizaje. El niño estará divirtiéndose y también aprendiendo acerca del teclado. Esta tarea es fundamental puesto que para ocupar la computadora el usuario debe conocer el teclado.

\section{Agradecimientos}

Queremos agradecer a los niños ciegos junto con sus padres quienes nos ayudaron para hacer las pruebas de usabilidad, a la Universidad de la Mixteca por facilitarnos el UsaLab para realizar dichas pruebas así como a los encargados del laboratorio y especialistas que estuvieron presentes. De la misma forma a profesores especialistas en esta área del CAM No. 21.

\section{Referencias}

[1] Flores Alvarado, Y. A., Mendoza García, R., Arriaga Fierro, A., Peralta Calvo, M., Hernández Paxtián, Z. J., \& Torales Iniesta, J. S. (2017). SAMM: System Designed to Help, Motivate and Improve the Experience of Visually Impaired People with Technology . México: ACM Digital Library.

[2] Gelman, D. L. (2014). Design For Kids. Brooklyn: Rosenfeld Media.
[3] Hassenzahl, M. (s.f.). Interaction Design foundation. Obtenido de User Experience and Experience Design: https://www.interaction-design.org/literature/book/theencyclopedia-of-human-computer-interaction-2nd-ed/userexperience-and-experience-design

[4] INEGI. 2017. Censo de Población y Vivienda 2010. Obtenido de Censo de Población y Vivienda 2010. Recuperado de http://www.inegi.org.mx/est/contenidos/proyectos/accesomic rodatos/cpv2010/ Mather, B.D. Making up titles for conference papers. Ext. Abstracts CHI 2000, ACM Press (2000), 1-2.

[5] Nielsen, J. (1993). Usability Engineering. Estados Unidos: Morgan Kaufmann.Zellweger, P.T., Bouvin, N.O., Jehøj, H., and Mackinlay, J.D. Fluid Annotations in an Open World. Proc. Hypertext 2001, ACM Press (2001), 9-18.

[6] Norman D., Draper S.1986. User Centered System Design: New Perspectives on Human-Computer Interaction. NJ, USA.

[7] Scrum.org. (s.f.). WHAT IS SCRUM? Obtenido de WHAT IS SCRUM?: https://www.scrum.org/resources/what-isscrum

[8] Stephanidis, C. (s.f.). Interaction Design Fundation. Obtenido de Design For All: https://www.interactiondesign.org/literature/book/the-encyclopedia-of-humancomputer-interaction-2nd-ed/design-4-all

[9] Steven, P. Dow. 2010. Wizard-of-Oz Prototyping. Stanford HCI Group 